Check for updates

Cite this: Mater. Chem. Front., 2019, 3, 2051

Received 20th June 2019, Accepted 29th July 2019

DOI: $10.1039 / \mathrm{c} 9 q m 00401 \mathrm{~g}$

rsc.li/frontiers-materials

\section{Bright electrochemiluminescent films of efficient aggregation-induced emission luminogens for sensitive detection of dopamine $\dagger$}

\author{
Zihua Li, ${ }^{a}$ Wei Qin, (D)*a Jialong Wu, ${ }^{a}$ Zhiyong Yang, (D) ${ }^{\mathrm{b}}$ Zhenguo $\mathrm{Chi}\left(\mathbb{D}^{\mathrm{b}}\right.$ and \\ Guodong Liang iD *a
}

\begin{abstract}
The development of electrochemiluminescent $(E C L)$ luminogens is of great importance for sensitive detection of biomolecules in various applications. Herein, two efficient red luminogens bearing benzothiadiazole and arylamino moieties, namely BTD-TPA and BTD-NPA, have been synthesized through one-step Suzuki reaction. They show aggregation-induced emission (AIE) features with high fluorescence quantum efficiency and reversible redox pairs with high stability. Taking advantage of these merits, bright ECL non-doped films are achieved based on the AIE luminogens. Interestingly, the ECL intensity of the films is proportional to the film thickness, which enables the optimization of their brightness through the variation of luminogen loading. Furthermore, the bright ECL films are utilized for sensitive detection of dopamine (DA) with a broad linear range $(0.05-350 \mu \mathrm{M})$ and a low detection limit of $17.0 \mathrm{nM}$. Such bright ECL films provide an ideal platform for sensitive analysis of important biomolecules with high selectivity.
\end{abstract}

\section{Introduction}

Electrochemiluminescence (ECL) has attracted enormous attention in immunoassay and analytical science due to its remarkable advantages, including simple instruments, low background, and high sensitivity. ${ }^{1-3}$ To date, different ECL systems based on metal complexes and quantum dots have been developed for in vitro diagnosis, DNA detection, ${ }^{4,5}$ and other biological applications. ${ }^{6-9}$ However, they encounter a few inherent problems such as high cost, heavy-metal toxicity, and poor processability. To circumvent the problems, organic fluorescent materials based on anthracene, fluorene, thienyltriazole, boron dipyrromethene (BODIPY), and pyridopyrimidine functional moieties have been developed for ECL applications due to their low cost, easy functionalization, and good biocompatibility. ${ }^{10-13}$ Most of the organic chromophores are constructed from hydrophobic aromatic moieties and used in solution. ${ }^{14}$ Compared with solution-based ECL systems, solid ECL films have overwhelming advantages, such as environmental friendliness,

\footnotetext{
${ }^{a}$ PCFM Lab, School of Materials Science and Engineering, Sun Yat-sen University, Guangzhou 510275, P. R. China.E-mail: qinw9@mail.sysu.edu.cn, lgdong@mail.sysu.edu.cn

${ }^{b}$ PCFM Lab, School of Chemistry, Sun Yat-sen University, Guangzhou 510275, P. R. China

$\dagger$ Electronic supplementary information (ESI) available: Reagents, experimental details, apparatus and characterization. Movies, UV and PL spectra, electrochemical data, ECL measurements, and quantitative detection. See DOI: 10.1039/ c9qm00401g
}

enhanced and stable ECL signal, and high cost-efficiency. ${ }^{15}$ However, conventional organic chromophores suffer from severe aggregation-caused quenching (ACQ) effect when used in solid films. Indeed, limited by the ACQ effect, efficient ECL films based on conventional organic chromophores are rarely reported. ${ }^{16}$ Although doping or labeling in polymers alleviates the ACQ effect, the chromophores are normally retained at low concentration $(<3 \mathrm{wt} \%)$ in the host materials, which greatly hinders their practical applications. ${ }^{17}$

The discovery of the aggregation-induced emission (AIE) phenomenon has opened a new avenue to solve the ACQ problem. ${ }^{18-20}$ Opposite to the ACQ effect, the fluorescence emission of AIE luminogens is enhanced by aggregate formation, which offers a new chance for the development of solid ECL films. ${ }^{21,22}$ A few of the efficient luminogens with AIE features have been developed and utilized in bioimaging and organic lightemitting diodes (OLEDs) due to their high brightness in the aggregated state. ${ }^{23-26}$ However, to our best knowledge, there is no report of efficient organic luminogens for bright ECL film applications, possibly due to their irreversible redox waves and poor stability. ${ }^{27-29}$

Dopamine (DA) is a significant neurotransmitter, which plays a key role in the function of the central nervous, hormonal, and cardiovascular systems. The abnormal concentration levels of DA are associated with some notorious diseases of the nervous system, such as Parkinson's, Alzheimer's and schizophrenia disease. ${ }^{30}$ Common methods for DA detection are based on high-performance liquid chromatography (HPLC) or mass 
spectrometry (MS). ${ }^{31,32}$ However, these methods are limited in practical applications due to expensive instruments, timeconsuming analysis, and laborious sample pretreatment. ${ }^{33}$ Recently, electrochemical methods based on fullerene, $\mathrm{ZnO}$ nanowires, and carbon nanotubes, were employed to detect DA with a detection limit in the range of 0.1-60 nM. ${ }^{34-40}$

Herein, we develop a type of bright ECL film based on efficient AIE luminogens (BTD-TPA and BTD-NPA). The AIE luminogens are constructed with a benzothiadiazole (BTD) core as an electron acceptor and an arylamino unit as an electron donor, ${ }^{41,42}$ giving rise to red luminogens through intramolecular charge transfer effect. Freely rotating rotors from the functional arylamino units endow them with AIE features. They show stable and reversible redox pairs inherited from the functional arylamino moieties. Taking advantage of the AIE features and reversible redox pairs, bright and tunable ECL films are achieved. Moreover, the bright ECL films are further utilized for sensitive detection of DA with a high Stern-Volmer constant $\left(5.5 \times 10^{5} \mathrm{M}^{-1}\right)$ and a low detection limit $(17.0 \mathrm{nM})$. The interference experiment demonstrates the sensitive detection of DA with good selectivity. Such bright ECL films provide a promising platform for rapid and quantitative analysis of bioassays with high sensitivity and selectivity.

\section{Experimental}

\section{Synthesis of BTD-Ph, BTD-TPA, and BTD-NPA}

The compounds were synthesized according to the procedure reported in the literature. ${ }^{43}$ The detailed synthetic procedure is presented in the ESI. $\dagger$

\section{Preparation of non-doped films}

The films were facilely prepared by drop-casting $5 \mu \mathrm{L}$ of DCM solution of luminogens on L-type GCE, followed by air drying. The film thickness is regulated by using luminogen solutions at various concentrations. The film thickness of BTD-TPA was measured using a fluorescence microscope (Leica M205 FA).

\section{Electrochemical and ECL measurements}

All electrochemistry experiments were performed with a threeelectrode cell on an electrochemical workstation (CHI 660D, Chenhua instrument, China). Glassy carbon electrodes (GCE, $3 \mathrm{~mm}$ diameter) and gold ultramicroelectrodes (UME, $12.5 \mu \mathrm{m}$ diameter) were employed as the working electrodes. Platinum wire was used as the counter electrode and silver wire was used as the quasi-reference electrode. An L-type GCE (3 mm diameter) was used as the working electrode for ECL experiments. Before usage, all working electrodes were polished with $0.5,0.3$, and $0.05 \mu \mathrm{m}$ alumina powder dispersed in water and sonicated in ethanol and deionized water. Before the end of each experiment, the $\mathrm{Ag}$ reference electrode was calibrated against ferrocene as an internal redox standard $\left(0.464 \mathrm{~V}\right.$ vs. SCE) ${ }^{44}$ Electrochemistry and ECL experiments were obtained in DCM and the ECL of BTD-TPA and BTD-NPA films were performed in $\mathrm{CH}_{3} \mathrm{CN} / \mathrm{H}_{2} \mathrm{O}$ $(\mathrm{v} / \mathrm{v}=7: 1)$ mixture with $0.1 \mathrm{M} \mathrm{TBAPF}_{6}$ as the supporting electrolyte. $20 \mathrm{mM}$ TPrA was used as a co-reactant in the ECL experiments of BTD-TPA and BTD-NPA, while $5 \mathrm{mM}$ BPO was employed as a co-reactant in the ECL experiment of BTD-Ph. A photomultiplier tube detector (PMT, Hamamatsu, Japan) with the voltage at $800 \mathrm{~V}$ was employed to collect the ECL signal. The ECL spectrum was obtained via pulsing $80 \mathrm{mV}$ past the peak potentials of the oxidation or reduction waves for BTD-TPA, BTD-NPA, and BTD-Ph with a pulse width of $1 \mathrm{~s}$ by differential pulse amperometry. The ECL intensity-time profiles were obtained by pulsing between $80 \mathrm{mV}$ past the oxidation peak and $0 \mathrm{~V}$ for BTD-TPA and BTD-NPA, and pulsing between $80 \mathrm{mV}$ past the reduction peak and $0 \mathrm{~V}$ for BTD-Ph by means of multi-potential steps, with a pulse width of $1 \mathrm{~s}$. The relative ECL efficiencies of BTD-Ph, BTD-TPA, and BTD-NPA in the solution state are calculated through integration of both ECL intensity and current value versus time with DPA $\left(\Phi_{\mathrm{ECL}}\right.$ is about $0.25 \%$ in $\left.\mathrm{DMF}^{11}\right)$ or $\left[\mathrm{Ru}(\mathrm{bpy})_{3}\right]^{2+}\left(\Phi_{\mathrm{ECL}}\right.$ is about $5.0 \%$ in acetonitrile $\left.{ }^{12}\right)$ as a standard (eqn (S1), ESI $\dagger$ ).

\section{Results and discussion}

BTD-TPA, BTD-NPA, and their counterpart (BTD-Ph) for comparison were facilely synthesized through one-step Suzuki coupling with satisfactory yields as shown in Scheme 1 (Fig. S1-S6, ESI $\dagger$ ). They possess good solubility in common organic solvents, such as toluene (Tol), chloroform, dichloromethane (DCM), 1,4-dioxane (DO), and tetrahydrofuran (THF), but are insoluble in acetonitrile or water. The UV spectra of BTD-TPA and BTD-NPA in THF are peaked at $458 \mathrm{~nm}$ with a large red-shift of $78 \mathrm{~nm}$ compared to BTD-Ph $(380 \mathrm{~nm})$ (Fig. S7, ESI $\dagger$ ). Compared to that of BTD-Ph, the fluorescence emission of BTD-TPA and BTD-NPA is more sensitive to solvent polarity (Fig. 1 and Fig. S8, Table S1, $\mathrm{ESI} \dagger$ ). All the results indicate that BTD-TPA and BTD-NPA demonstrate strong charge transfer effects from the electron-donating arylamino units to the electron-accepting benzothiadiazole core. ${ }^{45}$

To study the effects of aggregation and solvent polarity on the emission process, the photoluminescence (PL) spectra of BTD-Ph, BTD-TPA, and BTD-NPA were explored in THF/water mixtures with different water fractions $\left(f_{\mathrm{w}}\right)$. As shown in Fig. 2 and Fig. S9 (ESI $\dagger$ ), BTD-Ph emits green fluorescence with an emission maximum at $481 \mathrm{~nm}$ in pure THF. After the addition of water into THF, its PL intensity is decreased gradually. At $f_{\mathrm{w}}=90 \%$, the PL intensity is merely $15 \%$ of that in pure THF

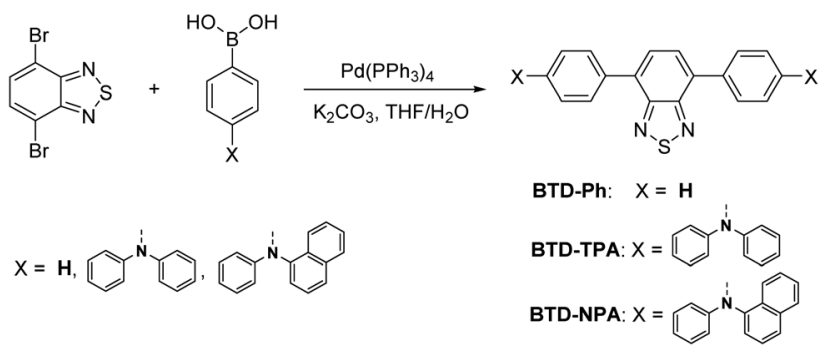

Scheme 1 Synthetic routes and chemical structures of BTD-Ph, BTD-TPA and BTD-NPA. 

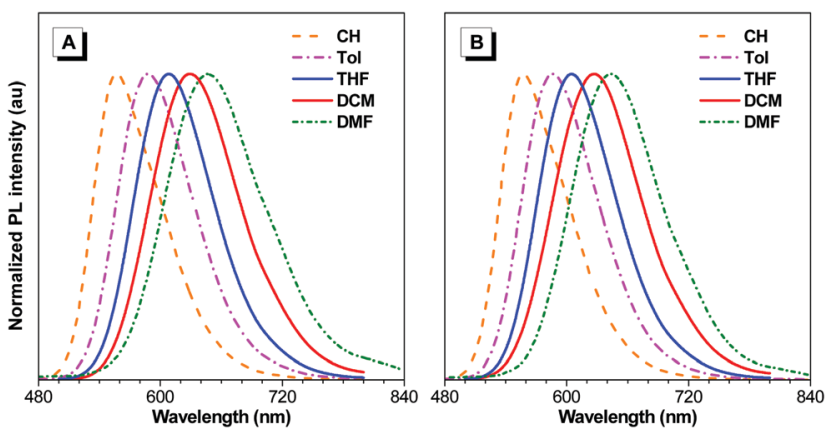

Fig. 1 Normalized PL emission spectra of (A) BTD-TPA, and (B) BTD-NPA in solvents with different polarities.
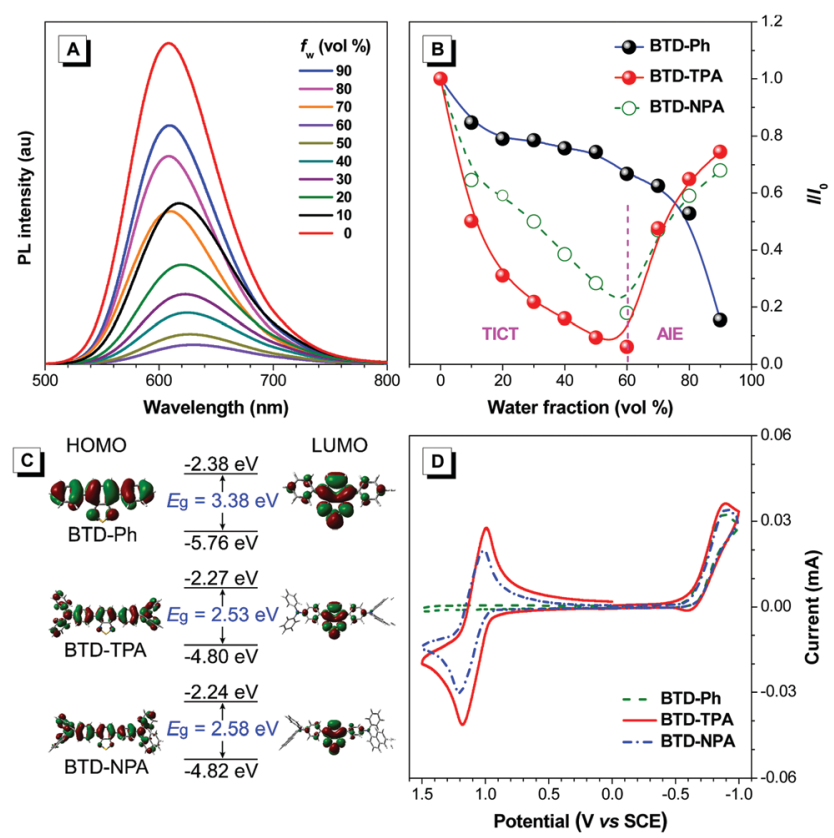

Fig. 2 (A) PL spectra of BTD-TPA in THF/water mixtures with different water fractions $\left(f_{\mathrm{w}}\right)$. (B) Plots of the relative $\mathrm{PL}$ intensity $\left(/ / /_{0}\right)$ versus the composition of the THF/water mixtures of BTD-Ph, BTD-TPA, and BTD-NPA. $I_{0}$ denotes emission intensity in pure THF. Concentration: $10 \mu \mathrm{M}$. (C) Optimized geometries and molecular orbitals of HOMO and LUMO levels of the molecules. (D) CVs of BTD-Ph, BTD-TPA, and BTD-NPA in anhydrous DCM.

solution accompanied by a $25 \mathrm{~nm}$ red-shift, indicative of the typical ACQ feature. In contrast, BTD-TPA and BTD-NPA in pure THF emit orange-red fluorescence with an emission maximum at 609 and $605 \mathrm{~nm}$, respectively. With the gradual addition of water into THF $\left(f_{\mathrm{w}} \leq 60 \%\right)$, the emission is weakened and redshifted to $c a .625 \mathrm{~nm}$ due to transformation to the twisted intramolecular charge transfer (TICT) state in mixed solvents. ${ }^{46}$ Afterwards $\left(f_{\mathrm{w}}>60 \%\right)$, the PL intensity starts to increase due to the formation of luminogen aggregates and the AIE effect from the freely rotating rotors of the arylamino units. The higher the water fraction, the stronger the emission intensity. At $f_{\mathrm{w}}=90 \%$, the PL intensity recovers remarkably, and the emission maximum moves to 609 and $606 \mathrm{~nm}$, respectively. BTD-TPA and BTD-NPA solids possess high fluorescence quantum yields ( $\Phi_{\mathrm{F}}$ ) of $44 \%$ and $56 \%$, respectively, which favors their application in solid films.

To study the photophysical properties of the compounds at the molecular level, density functional theory (DFT) calculations were carried out (Fig. 2C). The highest occupied molecular orbitals (HOMOs) of the studied molecules are dispersed on the whole molecules. While the electron clouds of the lowest unoccupied molecular orbitals (LUMO) are dominated on the BTD core and the adjacent benzene rings. The remarkable difference in electron distributions of the HOMO and LUMO levels of BTD-TPA and BTD-NPA indicates an intrinsic intramolecular charge transfer effect. Compared to the bandgap of BTD-Ph, the narrow band gaps of BTD-TPA and BTD-NPA are indicative of stronger electron donor-acceptor systems, which is consistent with the experimental data (Fig. S7-S9, ESI $\dagger$ ).

Cyclic voltammetry (CV) measurements were carried out to investigate the electrochemical behaviours of BTD-Ph, BTD-TPA, and BTD-NPA (Fig. 2D). BTD-TPA and BTD-NPA exhibit a stable and reversible redox pair at about $+1.1 \mathrm{~V}$ due to the presence of arylamine moieties. The anodic peak current $\left(i_{\mathrm{pa}}\right)$ and cathodic peak current $\left(i_{\mathrm{pc}}\right)$ are linearly dependent on the square root of the scan rate $\left(\nu^{1 / 2}\right)$ (Fig. S10-S12, ESI $\dagger$ ), demonstrating diffusion control in these redox processes. The peak current ratio $\left(i_{\mathrm{pa}} / i_{\mathrm{pc}}\right)$ of the redox pair of BTD-TPA and BTD-NPA is close to unity, revealing that radical cations generated during anodic potential scanning are stable. ${ }^{47}$ CVs of BTD-TPA and BTD-NPA using gold ultramicroelectrodes (UME) obtain similar results (Fig. S13, S14 (ESI $\dagger$ ), and Table 1). Such stable and reversible redox properties of BTD-TPA and BTD-NPA are essential for their further ECL applications.

The ECL properties of BTD-Ph, BTD-TPA, and BTD-NPA in DCM solution were investigated. In a co-reactant system, the ECL and corresponding CVs of all compounds were performed in DCM solution in the presence of benzoyl peroxide (BPO) or tripropylamine (TPrA) (Fig. S15, ESI $\dagger$ ). The bright ECL of BTD-Ph, BTD-TPA, and BTD-NPA can be observed by the naked eye with emission peaks at 493,633, and $632 \mathrm{~nm}$, respectively (Fig. S16, ESI $\dagger$ ). The ECL spectra resemble their PL spectra, which confirms that the ECL is from the luminogens. The ECL efficiencies of BTD-Ph, BTD-TPA, and BTD-NPA in solution are calculated to be $0.02 \%, 2.5 \%$, and $2.4 \%$, respectively. The ECL

Table 1 Electrochemical data

\begin{tabular}{|c|c|c|c|c|c|c|}
\hline \multirow[b]{2}{*}{ Compound } & \multicolumn{2}{|c|}{$E_{1 / 2}(\mathrm{~V} v s . \mathrm{SCE})^{a}$} & \multirow{2}{*}{$\begin{array}{l}10^{-6} D^{b} \\
{\left[\mathrm{~cm}^{2} \mathrm{~s}^{-1}\right]}\end{array}$} & \multirow{2}{*}{$\begin{array}{l}E_{\mathrm{g}}{ }^{c} \\
{[\mathrm{eV}]}\end{array}$} & \multirow{2}{*}{$\begin{array}{l}E_{\text {HOMO }}{ }^{d} \\
{[\mathrm{eV}]}\end{array}$} & \multirow[b]{2}{*}{$E_{\mathrm{LUMO}}{ }^{e}[\mathrm{eV}]$} \\
\hline & $\mathrm{A} / \mathrm{A}^{+a}$ & $\mathrm{~A} / \mathrm{A}^{-a}$ & & & & \\
\hline BTD-Ph & $\mathrm{nd}^{f}$ & -0.74 & $\mathrm{nd}^{f}$ & 2.87 & -5.74 & -2.87 \\
\hline BTD-TPA & 1.09 & -0.76 & 4.37 & 2.33 & -5.24 & -2.91 \\
\hline BTD-NPA & 1.11 & -0.77 & 14.3 & 2.33 & -5.27 & -2.94 \\
\hline
\end{tabular}

${ }^{a}$ The values of $E_{1 / 2}$ are obtained by averaging between the cathodic and anodic peak potentials. ${ }^{b} D=\pi a^{2} / 16 S^{2}, a$ is the radius of gold UME, $S$ is the slope of $i-t^{-1 / 2}$ in chronoamperometry. ${ }^{c}$ HOMO-LUMO band gap $\left(E_{\mathrm{g}}\right)$ is calculated from the onset of the absorption spectrum. ${ }^{d}$ HOMO is the highest occupied molecular orbital derived from the onset oxidation potential. ${ }^{e}$ LUMO is the lowest unoccupied molecular orbital estimated by using the equation $E_{\mathrm{LUMO}}=E_{\mathrm{HOMO}}+E_{\mathrm{g} .}{ }^{f}$ nd: not detected due to without oxidation wave of BTD-Ph. 


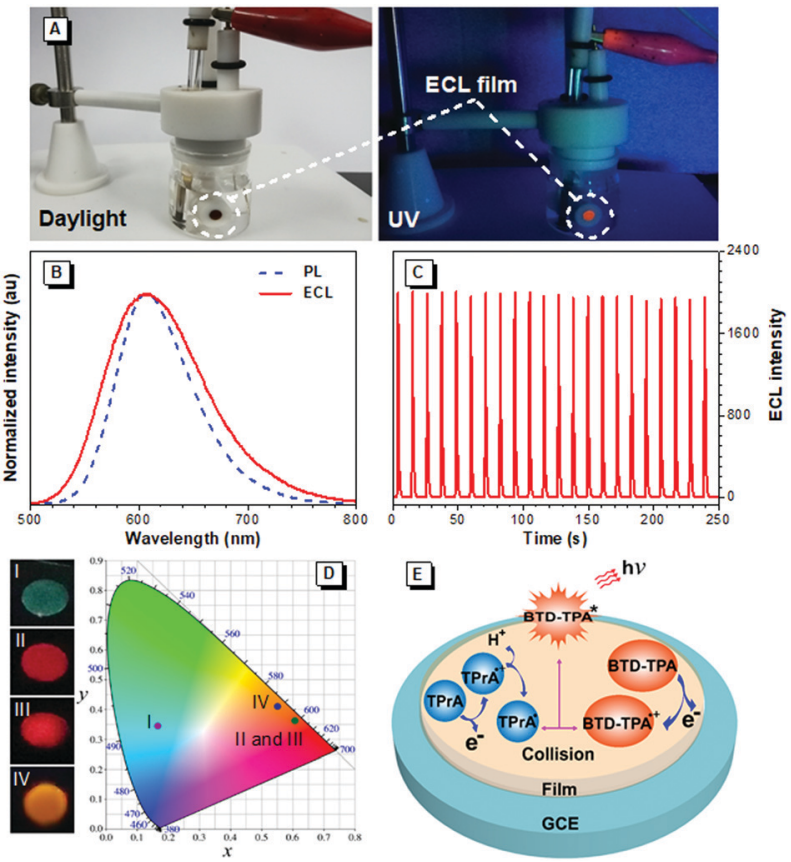

Fig. 3 (A) Digital photos of the experimental setup after the ECL experiment under daylight and UV illumination. (B) Normalized ECL and PL spectra of the BTD-TPA films. Excitation: $460 \mathrm{~nm}$. (C) ECL stability of the BTD-TPA films. (D) ECL photos of BTD-Ph (I), BTD-TPA (II), and BTD-NPA (III) in DCM solution, the BTD-TPA film (IV), and CIE chromaticity coordinates of their ECL spectra. (E) Proposed ECL mechanism of BTD-TPA films.

stabilities of BTD-Ph, BTD-TPA, and BTD-NPA were investigated by the pulsing technique (Fig. S17, ESI $\dagger$ ). BTD-TPA and BTD-NPA show good ECL stabilities and retain at an almost constant value during the consecutive cyclic potential scanning with a relative standard derivation (RSD) of $1.0 \%$ and $2.3 \%$, respectively. In contrast, the ECL signal of BTD-Ph is decreased with increasing scanning cycles. The decreased ECL signal of BTD-Ph is ascribed to the unstable radical anion, which greatly hinders the generation of the excited state of BTD-Ph. ${ }^{10}$

Encouraged by their promising solution ECL results and AIE features, we developed solid ECL films based on BTD-TPA and BTD-NPA (Fig. 3A). The films were facilely prepared by dropcasting DCM solution of the luminogens on a glassy carbon electrode (GCE). The solid films of BTD-TPA and BTD-NPA turn on at low voltages of $+0.80 \mathrm{~V}$ and $+0.65 \mathrm{~V}$, respectively, which are much lower than that of $\mathrm{Ru}(\mathrm{bpy})_{3}{ }^{2+}(+1.2 \mathrm{~V}) .^{48}$ The ECL intensities of BTD-TPA and BTD-NPA reach the maximum values at $+1.05 \mathrm{~V}$ and $+1.14 \mathrm{~V}$, respectively (Fig. S18-S20, ESI $\dagger$ ). The ECL spectrum of the BTD-TPA film resembles its PL spectrum (Fig. 3B). The ECL quantum efficiency of BTD-TPA films is calculated to be $4.0 \%$, using $\mathrm{Ru}(\mathrm{bpy})_{3}{ }^{2+}$ as a reference with the ECL quantum efficiency of $5.0 \%$ (eqn (S1), ESI $\dagger$ ). The ECL signal of BTD-TPA films remains almost at a constant value during consecutive cyclic potential scanning with an RSD of 1.4\% (Fig. 3C). In contrast, the ECL intensity of BTD-NPA films gradually decreases to $36.5 \%$ of its maximum value after 27 cycles (Fig. S21, ESI $\dagger$ ) due to the formation of a thin polymer film on the electrode surface. ${ }^{13}$ The corresponding Commission Internationale de l'Eclairage (CIE) coordinates of BTD-Ph (I), BTD-TPA (II), and BTD-NPA (III) in DCM solution are calculated to be $(0.17,0.35)$, $(0.62,0.36)$, and $(0.62,0.36)$, respectively. While the CIE coordinates of the spectrum of BTD-TPA solid film are calculated to be $(0.56,0.41)$, as shown in Fig. 3D. The ECL of BTD-TPA films is explained through an oxidative-reduction co-reactant mechanism (Fig. 3E). Both BTD-TPA and co-reactant TPrA are first oxidized to the corresponding radical cation BTD-TPA $^{\bullet+}$ and intermediate radical cation $\operatorname{TPrA}^{\bullet+}$. TPrA ${ }^{\bullet+}$ loses a proton to generate the neutral radical $\operatorname{TPrA}^{\bullet}$ on the electrode surface. Then BTD-TPA $^{{ }^{+}}$is reduced by $\operatorname{TPrA}^{\bullet}$ to form excited-state BTD-TPA $^{*}$, which finally emits light and returns to its ground state (eqn (S2)-(S6), ESI $\dagger$ ). ${ }^{49,50}$

The brightness of the ECL films is tunable. The ECL intensity of the BTD-TPA films is remarkably enhanced with the increase in the luminogen loading from $25 \mathrm{ng} \mathrm{mm} \mathrm{mm}^{-2}$ to $1 \mu \mathrm{g} \mathrm{mm}$ (Fig. 4). The thickness of the solid films was further measured using a fluorescence microscope. Interestingly, the ECL intensity of the BTD-TPA films with increasing thickness demonstrates a linear relationship $(R=0.9973)$. The ECL intensity of the films is enhanced by 58 -fold when the film thickness is increased from $26 \mu \mathrm{m}$ to $130 \mu \mathrm{m}$. Evidently, the ECL intensity of BTD-TPA films is tunable through facilely regulating luminogen loading or film thickness, which is useful for their practical applications. Such tunable ECL intensity of BTD-TPA films benefits from AIE features of the luminogen. The current during ECL of BTD-TPA films was monitored. The current increases linearly with increasing luminogen loading (Fig. S22, ESI $\dagger$ ), suggesting that BTD-TPA molecules in the films involve ECL reactions.

To investigate the morphologies and microstructure of BTD-TPA films, scanning electron microscopy (SEM) and X-ray diffraction (XRD) were performed. As shown in Fig. S23 (ESI $\dagger$ ), continous and dense films are observed. BTD-TPA films have a smooth surface. No ordered sub-structures are recognized in the films. Moreover, the XRD spectrum displays that the diffraction peaks of BTD-TPA film are in good agreement with the simulated peak from single crystals, ${ }^{45}$ confirming the formation of a crystal

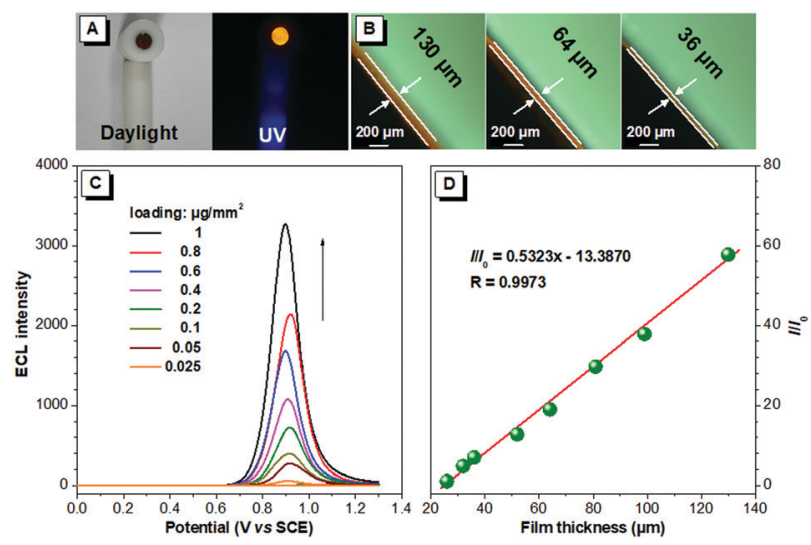

Fig. 4 (A) Digital photos of BTD-TPA film under daylight and UV illumination. (B) Optical microscope images of BTD-TPA films with different thicknesses. (C) ECL intensity of BTD-TPA films with various loadings. (D) Plot of relative $E C L$ intensity $\left(I / I_{0}\right)$ versus the different thickness of BTD-TPA films; $I_{0}$ denotes the ECL emission intensity of BTD-TPA film with a thickness of $26 \mu \mathrm{m}$. 
structure in BTD-TPA films (Fig. S24, ESI $\dagger$ ). Furthermore, electrochemical impedance spectroscopy (EIS) was employed to monitor the electrochemical properties of BTD-TPA films at various loadings. The semicircle diameter at higher frequencies reflects the electron-transfer resistance $\left(R_{\mathrm{ct}}\right)$, which is related to the electron-transfer kinetics. The bare GCE displays a small $R_{\text {ct }}$ value (225 $\Omega$, Fig. S25, ESI $\dagger$ ). Modification of the GCE with BTD-TPA at $0.1 \mu \mathrm{g} \mathrm{mm}{ }^{-2}$ leads to a 5 -fold $R_{\text {ct }}$ value $(1022 \Omega)$. With increasing BTD-TPA loading, the $R_{\mathrm{ct}}$ value increases. The GCE modified with $1 \mu \mathrm{g} \mathrm{mm} \mathrm{mm}^{-2}$ of BTD-TPA shows an $R_{\mathrm{ct}}$ value of $2199 \Omega$. The moderate $R_{\mathrm{ct}}$ values of the BTD-TPA modified GCE in the order of $\mathrm{k} \Omega$ show an acceptable electrical conductivity of BTD-TPA films and efficient electrical communication among the GCE, BTD-TPA films, and supporting electrolyte.

In ECL films, a small amount of luminogens (typically $50 \mathrm{ng}$ to $14 \mu \mathrm{g}$ ) is used. ${ }^{16}$ In contrast, much higher amounts of luminogens by two orders of magnitude are required in solution ECL systems (typically $2.0 \mathrm{mg}$ ). ${ }^{10,51}$ Thus, the present ECL films greatly reduce the consumption of luminogens. Moreover, in ECL films, luminogens are loaded on the electrode surface. Poor solvents of luminogens such as water and acetonitrile are used as supporting liquid. A large volume of toxic and volatile solvents such as DCM is required in solution ECL systems. Evaporation of the toxic solvents during ECL measurements causes not only unstable ECL but also environmental issues. Thus, the ECL films are a promising platform for further applications. In recent years, there have been a few reports of immobilization of metal complexes on the electrode surface for ECL through the Langmiur-Blodegtt method or self-assembly ${ }^{15}$ However, the immobilization methods are far from satisfactory due to the problems from unstable biased potentials and easy desorption of luminophores from solid electrodes. Although a series of approaches for ECL enhancement through the preparation of modified electrodes are reported, ${ }^{52-54}$ they involve complicated modification processes on electrodes. This greatly hinders their practical applications. Thus, the present work using inexpensive organic AIE luminogens demonstrates a simple and direct approach for the development of bright and tunable ECL devices without involving any ancillary methods.

With the merits of bright ECL and superior stability, BTD-TPA films were used for the detection of dopamine (DA). The blank BTD-TPA film emits strong ECL with an emission maximum at $607 \mathrm{~nm}$. The ECL signal of BTD-TPA films is stable and reproducible. ECL intensity changes rarely during consecutive scanning (Fig. S26, ESI $\dagger$ ). The ECL intensity of BTD-TPA films is gradually quenched with increasing concentration of DA. When the concentration of DA is increased to $350 \mu \mathrm{M}$, the ECL intensity of BTD-TPA thin film is almost quenched with only $<4 \%$ of its initial ECL intensity (Fig. 5A). ECL quenching of BTD-TPA films follows the Stern-Volmer equation at low DA concentration. ${ }^{55}$

$$
I_{0} / I=K[\mathrm{Q}]+1
$$

where $I_{0}$ and $I$ are the ECL intensities in the absence and presence of a quencher, respectively. $K$ denotes the Stern-Volmer constant in the unit of $\mathrm{M}^{-1}$, which represents the sensitivity of

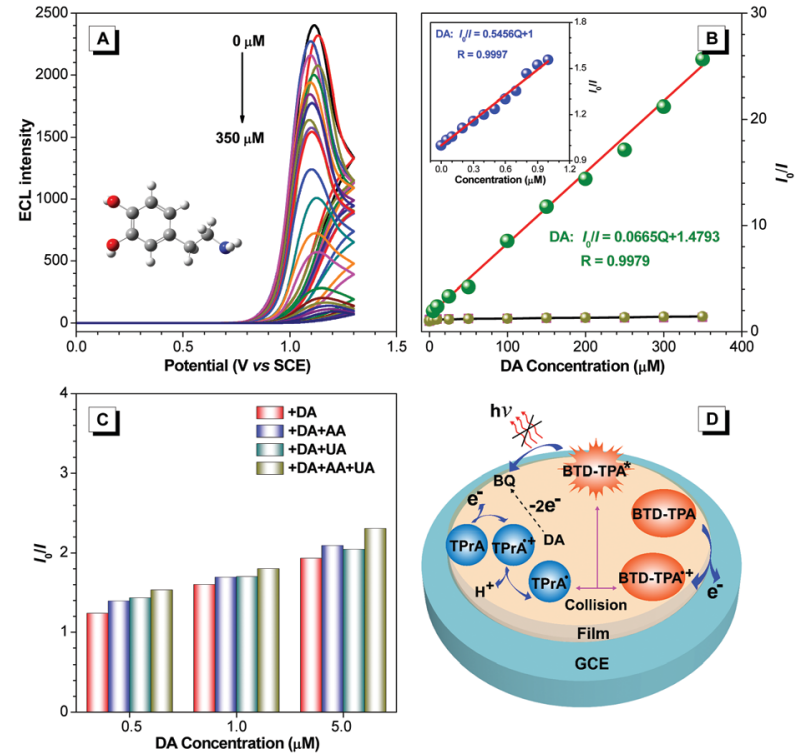

Fig. 5 (A) ECL intensity versus potential profiles of BTD-TPA film with different concentrations of DA. (B) Stern-Volmer plots of $I_{0} / /$ of BTD-TPA film versus the concentration of DA, AA, and UA. Inset: Stern-Volmer plot at low concentration of DA $(0-1 \mu \mathrm{M})$. (C) Io// of BTD-TPA film with different concentrations of DA containing 100-fold concentrations of AA and UA. (D) Proposed ECL quenching mechanism of the BTD-TPA film modified GCE.

the luminogen toward the quencher. A larger $K$ value suggests higher sensitivity toward the quencher. $[\mathrm{Q}]$ is the concentration of the quencher in the unit of $\mathrm{M}$. The plot is composed of two regions (Fig. 5B). In region I with DA concentration $\leq 1 \mu \mathrm{M}$, the plot demonstrates a linear relationship with a $K_{\mathrm{I}}$ value of $5.5 \times 10^{5} \mathrm{M}^{-1}$. The detection limit is calculated as low as $17.0 \mathrm{nM}$ (eqn (S7), ESI $\dagger$ ). When the DA concentration is increased from 1 to $350 \mu \mathrm{M}$, the Stern-Volmer plot is deviated from the initial linear relationship in region $I$ and follows another linear relationship with a $K_{\mathrm{II}}$ value of $6.6 \times 10^{4} \mathrm{M}^{-1}$. For comparison, the quenching properties of AA and UA were also investigated (Fig. 5B and Fig. S27, ESI $\dagger$ ). The quenching constants of ascorbic acid (AA) and uric acid (UA) are lowered by three orders of magnitude compared to that of DA $\left(9.0 \times 10^{2} \mathrm{M}^{-1}\right.$ and $5.0 \times 10^{2} \mathrm{M}^{-1}$, respectively), suggesting higher sensitivity toward DA. The interference of AA and UA on the detection process was further tested. Even containing an excessive amount of AA and UA (100-fold of DA), similar values of $I_{0} / I$ were obtained, further demonstrating high specificity of the ECL films toward DA (Fig. 5C and Fig. S28, ESI $\dagger$ ). The ECL quenching mechanism is proposed (Fig. 5D). DA is electrochemically oxidized into quinone species such as $o$-benzoquinone (BQ) during the ECL process, ${ }^{56,57}$ which consumes the energy of the excited state of BTD-TPA. The proposed mechanism is supported by the antioxidation experiment (Fig. 6). Indeed, upon addition of antioxidant 2-mercapoethanol ( $\beta \mathrm{ME}$ ) into the detection solution, the quenched ECL emission of the BTD-TPA film is greatly recovered. ${ }^{57}$

Thus, the ECL films in this work offer a promising platform for specific detection of DA. Recently, there have been a few reports of DA detection using different modified electrodes (Fig. 7 and Table S2, ESI $\dagger$ ). ${ }^{58-73}$ Most of the reported methods 


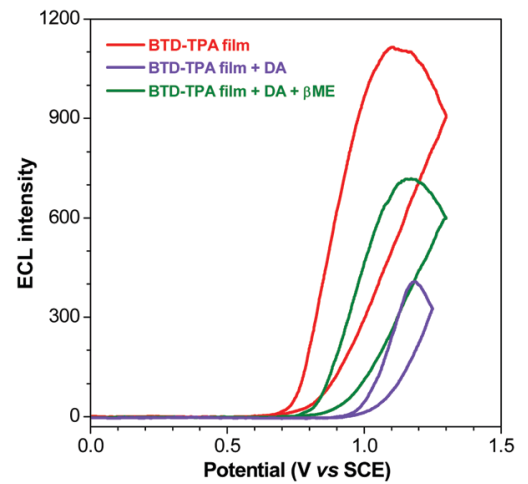

Fig. 6 ECL curves of the BTD-TPA film (red line), $+5 \mu \mathrm{M}$ DA (violet line) and $+100 \mu \mathrm{M} \beta M E$ (green line).

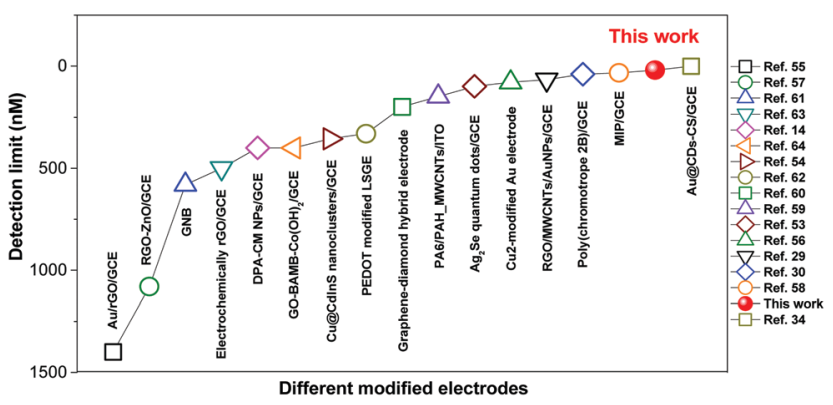

Fig. 7 Summary of detection limits for DA using different modified electrodes in the literature.

have detection limits from $33 \mathrm{nM}$ to $1400 \mathrm{nM}$. Moreover, relevant quenching constants are rarely reported. ${ }^{58,60}$ Compared to previous reports, the present results are interesting with a high quenching constant $\left(5.5 \times 10^{5} \mathrm{M}^{-1}\right)$, a wider linear range $(0.05-350 \mu \mathrm{M})$, and a lower detection limit (17.0 nM).

\section{Conclusions}

In summary, we have constructed two red luminogens from benzothiadiazole (BTD) and arylamino units, abbreviated as BTD-TPA and BTD-NPA, through one-step Suzuki reaction. The resultant molecules show AIE effects with high fluorescence efficiency and reversible redox pairs with high stability. Bright ECL solid films are fabricated based on the efficient AIE luminogens. Interestingly, the ECL intensity of the non-doped films is proportional to the film thickness, which allows tuning their brightness through facile variation of luminogen loading. Moreover, the bright ECL films are utilized for sensitive detection of dopamine (DA). A high Stern-Volmer constant $\left(5.5 \times 10^{5} \mathrm{M}^{-1}\right)$ and a low detection limit of $17.0 \mathrm{nM}$ are achieved with good selectivity. Such bright ECL films open a promising avenue for rapid and quantitative analysis of bioassays with high sensitivity and selectivity.

\section{Conflicts of interest}

There are no conflicts to declare.

\section{Acknowledgements}

The financial support is partially from NSFC (21374136), and the Fundamental Research Funds for the Central Universities (17lgjc03 and 18lgpy04).

\section{Notes and references}

1 M. M. Richter, Chem. Rev., 2004, 104, 3003-3036.

2 L. $\mathrm{Hu}$ and G. Xu, Chem. Soc. Rev., 2010, 39, 3275-3304.

3 L. Li, Y. Chen and J. J. Zhu, Anal. Chem., 2017, 89, 358-371.

4 W. Cao, J. P. Ferrance, J. Demas and J. P. Landers, J. Am. Chem. Soc., 2006, 128, 7572-7578.

5 G. Jie, H. Huang, X. Sun and J. J. Zhu, Biosens. Bioelectron., 2008, 23, 1896-1899.

6 S. Liu, H. Yuan, H. Bai, P. Zhang, F. Lv, L. Liu, Z. Dai, J. Bao and S. Wang, J. Am. Chem. Soc., 2018, 140, 2284-2291.

7 G. Valenti, S. Scarabino, B. Goudeau, A. Lesch, M. Jović, E. Villani, M. Sentic, S. Rapino, S. Arbault, F. Paolucci and N. Sojic, J. Am. Chem. Soc., 2017, 139, 16830-16837.

8 S. Voci, B. Goudeau, G. Valenti, A. Lesch, M. Jović, S. Rapino, F. Paolucci, S. Arbault and N. Sojic, J. Am. Chem. Soc., 2018, 140, 14753-14760.

9 P. Bertoncello and R. J. Forster, Biosens. Bioelectron., 2009, 24, 3191-3200.

10 R. Zhang, F. Tong, L. Yang, J. R. Adsetts, T. Yan, R. Wang, Z. Ding and H. B. Wang, Chem. Commun., 2018, 54, 9897-9900.

11 F. Rizzo, F. Polo, G. Bottaro, S. Fantacci, S. Antonello, L. Armelao, S. Quici and F. Maran, J. Am. Chem. Soc., 2017, 139, 2060-2069.

12 H. Qi, J. J. Teesdale, R. C. Pupillo, J. Rosenthal and A. J. Bard, J. Am. Chem. Soc., 2013, 135, 13558-13566.

13 F. Polo, F. Rizzo, M. Veiga Gutierrez, L. De Cola and S. Quici, J. Am. Chem. Soc., 2012, 134, 15402-15409.

14 H. Qi, Y. H. Chen, C. H. Cheng and A. J. Bard, J. Am. Chem. Soc., 2013, 135, 9041-9049.

15 Z. Guo and S. Dong, Anal. Chem., 2004, 76, 2683-2688.

16 M. Shen, X. H. Zhu and A. J. Bard, J. Am. Chem. Soc., 2013, 135, 8868-8873.

17 Z. Wang, S. Chen, J. W. Y. Lam, W. Qin, R. T. K. Kwok, N. Xie, Q. Hu and B. Z. Tang, J. Am. Chem. Soc., 2013, 135, 8238-8245.

18 J. Mei, N. L. C. Leung, R. T. K. Kwok, J. W. Y. Lam and B. Z. Tang, Chem. Rev., 2015, 115, 11718-11940.

19 J. Mei, Y. Huang and H. Tian, ACS Appl. Mater. Interfaces, 2018, 10, 12217-12261.

20 C. Wang and Z. Li, Mater. Chem. Front., 2017, 1, 2174-2194. 21 X. Wei, M. J. Zhu, Z. Cheng, M. Lee, H. Yan, C. Lu and J. J. Xu, Angew. Chem., 2019, 131, 3194-3198.

22 Z. Han, Z. Yang, H. Sun, Y. Xu, X. Ma, D. Shan, J. Chen, S. Huo, Z. Zhang, P. Du and X. Lu, Angew. Chem., 2019, 131, 5976-5980.

23 W. Qin, P. Zhang, H. Li, J. W. Y. Lam, Y. Cai, R. T. K. Kwok, J. Qian, W. Zheng and B. Z. Tang, Chem. Sci., 2018, 9, 2705-2710. 
24 W. Qin, Z. Yang, Y. Jiang, J. W. Y. Lam, G. Liang, H. S. Kwok and B. Z. Tang, Chem. Mater., 2015, 27, 3892-3901.

25 X. Han, B. Zhang, J. Chen, S. H. Liu, C. Tan, H. Liu, M. J. Lang, Y. Tan, X. Liu and J. Yin, J. Mater. Chem. B, 2017, 5, 5096-5100.

26 X. Y. Wang, J. Zhang, Y. B. Dong, Y. Zhang, J. Yin and S. H. Liu, Dyes Pigm., 2018, 156, 74-81.

27 L. Chen, Y. Jiang, H. Nie, P. Lu, H. H. Y. Sung, I. D. Williams, H. S. Kwok, F. Huang, A. Qin, Z. Zhao and B. Z. Tang, Adv. Funct. Mater., 2014, 24, 3621-3630.

28 Y. Liu, S. Chen, J. W. Y. Lam, P. Lu, R. T. K. Kwok, F. Mahtab, H. S. Kwok and B. Z. Tang, Chem. Mater., 2011, 23, 2536-2544.

29 Y. Liu, Y. Lv, H. Xi, X. Zhang, S. Chen, J. W. Y. Lam, R. T. K. Kwok, F. Mahtab, H. S. Kwok, X. Tao and B. Z. Tang, Chem. Commun., 2013, 49, 7216-7218.

30 S. R. Ali, Y. Ma, R. R. Parajuli, Y. Balogun, W. Y. C. Lai and H. He, Anal. Chem., 2007, 79, 2583-2587.

31 C. Muzzi, E. Bertocci, L. Terzuoli, B. Porcelli, I. Ciari, R. Pagani and R. Guerranti, Biomed. Pharmacother., 2008, 62, 253-258.

32 V. Carrera, E. Sabater, E. Vilanova and M. A. Sogorb, J. Chromatogr. B: Anal. Technol. Biomed. Life Sci., 2007, 847, 88-94.

33 F. Shang, L. Zhou, K. A. Mahmoud, S. Hrapovic, Y. Liu, H. A. Moynihan, J. D. Glennon and J. H. T. Luong, Anal. Chem., 2009, 81, 4089-4098.

34 Z. Su, Y. Liu, Q. Xie, L. Chen, Y. Zhang, Y. Meng, Y. Li, Y. Fu, M. Ma and S. Yao, Biosens. Bioelectron., 2012, 36, 154-160.

35 L. Wei, Y. Lei, H. Fu and J. Yao, ACS Appl. Mater. Interfaces, 2012, 4, 1594-1600.

36 H. Y. Yue, S. Huang, J. Chang, C. Heo, F. Yao, S. Adhikari, F. Gunes, L. C. Liu, T. H. Lee, E. S. Oh, B. Li, J. J. Zhang, T. Q. Huy, N. V. Luan and Y. H. Lee, ACS Nano, 2014, 8, 1639-1646.

37 H. Muguruma, Y. Inoue, H. Inoue and T. Ohsawa, J. Phys. Chem. C, 2016, 120, 12284-12292.

38 L. Jiang, G. W. Nelson, J. Abda and J. S. Foord, ACS Appl. Mater. Interfaces, 2016, 8, 28338-28348.

39 F. Wu, T. Huang, Y. Hu, X. Yang, Y. Ouyang and Q. Xie, Microchim. Acta, 2016, 183, 2539-2546.

40 W. Deng, X. Yuan, Y. Tan, M. Ma and Q. Xie, Biosens. Bioelectron., 2016, 85, 618-624.

41 J. Chen, F. Ye, Y. Lin, Z. Chen, S. Liu and J. Yin, Sci. China: Chem., 2019, 62, 440-450.

42 J. Chen, D. Li, W. Chi, G. Liu, S. H. Liu, X. Liu, C. Zhang and J. Yin, Chem. - Eur. J., 2018, 24, 3671-3676.

43 K. Shin Ichiro, M. Taisuke, S. Motoyuki, G. Hideki, M. Shuuichi, I. I. Tsutomu and M. Shuntaro, Chem. - Eur. J., 2010, 12, 2303-2317.

44 S. Sahami and M. J. Weaver, J. Electroanal. Chem. Interfacial Electrochem., 1981, 122, 155-170.

45 S. i. Kato, T. Matsumoto, T. Ishi, T. Thiemann, M. Shigeiwa, H. Gorohmaru, S. Maeda, Y. Yamashita and S. Mataka, Chem. Commun., 2004, 2342-2343.

46 W. Qin, D. Ding, J. Liu, W. Z. Yuan, Y. Hu, B. Liu and B. Z. Tang, Adv. Funct. Mater., 2012, 22, 771-779.
47 J. Suk, Z. Wu, L. Wang and A. J. Bard, J. Am. Chem. Soc., 2011, 133, 14675-14685.

48 M. Mayer, S. Takegami, M. Neumeier, S. Rink, A. Jacobi von Wangelin, S. Schulte, M. Vollmer, A. G. Griesbeck, A. Duerkop and A. J. Baeumner, Angew. Chem., Int. Ed., 2018, 57, 408-411.

49 R. Y. Lai and A. J. Bard, J. Phys. Chem. A, 2003, 107, 3335-3340.

50 W. Miao, J. P. Choi and A. J. Bard, J. Am. Chem. Soc., 2002, 124, 14478-14485.

51 K. N. Swanick, S. Ladouceur, E. Zysman Colman and Z. Ding, Chem. Commun., 2012, 48, 3179-3181.

52 B. Wu, C. Miao, L. Yu, Z. Wang, C. Huang and N. Jia, Sens. Actuators, B, 2014, 195, 22-27.

53 Y. P. Dong, G. Chen, Y. Zhou and J. J. Zhu, Anal. Chem., 2016, 88, 1922-1929.

54 Y. Yu, C. Lu and M. Zhang, Anal. Chem., 2015, 87, 8026-8032. 55 O. Stern and M. Volmer, Phys. Z., 1919, 20, 183-188.

56 J. McCall, C. Alexander and M. M. Richter, Anal. Chem., 1999, 71, 2523-2527.

57 X. Liu, H. Jiang, J. Lei and H. Ju, Anal. Chem., 2007, 79, 8055-8060.

58 H. Liu, L. Wang, H. Gao, H. Qi, Q. Gao and C. Zhang, ACS Appl. Mater. Interfaces, 2017, 9, 44324-44331.

59 D. Yuan, S. Chen, R. Yuan, J. Zhang and X. Liu, Sens. Actuators, B, 2014, 191, 415-420.

60 R. Cui, Y. P. Gu, L. Bao, J. Y. Zhao, B. P. Qi, Z. L. Zhang, Z. X. Xie and D. W. Pang, Anal. Chem., 2012, 84, 8932-8935.

61 F. Wang, J. Lin, H. Wang, S. Yu, X. Cui, A. Ali, T. Wu and Y. Liu, Nanoscale, 2018, 10, 15932-15937.

62 X. B. Li, M. M. Rahman, G. R. Xu and J. J. Lee, Electrochim. Acta, 2015, 173, 440-447.

63 C. Wang, J. Du, H. Wang, C. E. Zou, F. Jiang, P. Yang and Y. Du, Sens. Actuators, B, 2014, 204, 302-309.

64 G. Jiang, X. Gu, G. Jiang, T. Chen, W. Zhan and S. Tian, Sens. Actuators, B, 2015, 209, 122-130.

65 X. Zhang, Y. C. Zhang and L. X. Ma, Sens. Actuators, B, 2016, 227, 488-496.

66 M. Zhong, Y. Teng, S. Pang, L. Yan and X. Kan, Biosens. Bioelectron., 2015, 64, 212-218.

67 L. A. Mercante, A. Pavinatto, L. E. O. Iwaki, V. P. Scagion, V. Zucolotto, O. N. Oliveira, L. H. C. Mattoso and D. S. Correa, ACS Appl. Mater. Interfaces, 2015, 7, 4784-4790.

68 Q. Yuan, Y. Liu, C. Ye, H. Sun, D. Dai, Q. Wei, G. Lai, T. Wu, A. Yu, L. Fu, K. W. A. Chee and C. T. Lin, Biosens. Bioelectron., 2018, 111, 117-123.

69 P. K. Kannan, S. A. Moshkalev and C. S. Rout, Nanotechnology, 2016, 27, 075504.

70 Q. Huang, H. Zhang, S. Hu, F. Li, W. Weng, J. Chen, Q. Wang, Y. He, W. Zhang and X. Bao, Biosens. Bioelectron., 2014, 52, 277-280.

71 G. Xu, Z. A. Jarjes, V. Desprez, P. A. Kilmartin and J. T. Sejdic, Biosens. Bioelectron., 2018, 107, 184-191.

72 L. Yang, D. Liu, J. Huang and T. You, Sens. Actuators, B, 2014, 193, 166-172.

73 A. Ejaz, Y. Joo and S. Jeon, Sens. Actuators, B, 2017, 240, 297-307. 